\title{
Préparation du Canada en cas de grippe pandémique : stratégie relative aux mesures de santé publique
}

\author{
B Henry ${ }^{1}$ au nom du Groupe de travail sur la préparation du Canada en cas de grippe pandémique
}

\section{Résumé}

Les mesures de santé publique, ou interventions non pharmacologiques, sont des mesures de base cherchant à ralentir la propagation d'une maladie transmissible dans la collectivité. En cas de grippe pandémique, les mesures de santé publique et les médicaments antiviraux sont les seuls outils accessibles pour atténuer les effets de la pandémie au cours des mois précédant la création d'un vaccin. Le document Préparation du Canada en cas de grippe pandémique :Guide de planification pour le secteur de la santé (PCGP) indique comment les gouvernements fédéral, provinciaux et territoriaux collaboreront pour veiller à l'adoption d'une approche coordonnée et uniforme en matière de préparation et d'intervention en cas de grippe pandémique dans le secteur de la santé.

Le présent article résume la stratégie relative aux mesures de santé publique en cas de pandémie au Canada, comme l'indique l'annexe du document PCGP réservée aux mesures de santé publique récemment mise à jour. La stratégie s'appuie sur les leçons retenues lors de la pandémie de grippe H1N1 en 2009. Parmi les principaux éléments de la stratégie relative aux mesures de santé publique, il y a des mesures individuelles (p. ex. I'hygiène des mains, l'auto-isolement lors de la présence de la maladie), des mesures communautaires (p. ex. la fermeture d'écoles, l'annulation des rassemblements de masse), la gestion des cas et des contacts étroits, les mesures relatives aux déplacements et à la frontière, ainsi que l'éducation du public. Parmi les facteurs qui influent sur l'efficacité des mesures de santé publique en présence d'une pandémie, il y a l'épidémiologie de la pandémie, le moment de la mise en œuvre, l'évolutivité, la souplesse et la manière dont les mesures sont appliquées (seules ou en combinaison avec d'autres), ainsi que la conformité du public. Le document PCGP est un document d'orientation évolutif. L'annexe sera mise à jour dès que la publication de nouveaux renseignements l'exigera.
Cette oeuvre est mise à la disposition selon les termes de la licence internationale Creative Commons Attribution 4.0

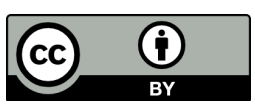

Affiliation

${ }^{1}$ Bureau de médecin-hygiéniste provincial, Victoria (Colombie-Britannique)

\section{*Correspondance :}

phac.cpip.tg.secretariat-gt.pcp. aspc@canada.ca

Citation proposée : Henry B au nom du Groupe de travail sur la préparation du Canada en cas de grippe pandémique. Préparation du Canada en cas de grippe pandémique : stratégie relative aux mesures de santé publique. Relevé des maladies transmissibles au Canada 2019;45(6):175-80.

https://doi.org/10.14745/ccdr.v45i06a03f

Mots-clés : grippe, pandémie, mesures de santé publique, interventions non pharmacologiques

\section{Introduction}

Le Canada dispose d'une approche à facettes multiples en matière de préparation et d'intervention en cas de grippe pandémique, qui comprend des mesures de santé publique. Ces mesures représentent les principales mesures non pharmacologiques servant à ralentir le taux de transmission virale. Elles sont mises en œuvre au début d'une pandémie, avant qu'un vaccin soit offert. Parmi les mesures de santé publique, il y a les mesures de protection individuelles (p. ex. I'hygiène des mains, l'auto-isolement en présence de la maladie), les mesures communautaires (p. ex. la fermeture des écoles, l'annulation des rassemblements de masse) et les mesures associées à la frontière 
internationale et aux déplacements (p. ex. un contrôle à l'arrivée et au départ).

La grippe pandémique H1N1 en 2009 a permis au Canada de mettre à l'essai, pour la première fois, ses efforts de planification en cas de pandémie. Il a également été confirmé que les mesures de santé publique constituaient un élément efficace et important d'intervention à la pandémie. On souligne que les mesures de santé publique sont un élément efficace et important d'une intervention en cas de pandémie. En outre, de nombreuses leçons utiles ont été retenues en ce qui concerne la planification et la mise en œuvre de mesures de santé publique, comme l'importance de mettre en application, de manière ciblée et graduelle, des mesures au début de la pandémie, et le fait de fournir des messages clairs et uniformes au public pour appuyer le respect des mesures recommandées.

L'annexe sur les mesures de santé publique du document Préparation du Canada en cas de grippe pandémique : Guide de planification pour le secteur de la santé $(1,2)$ décrit l'approche renouvelée concernant l'application de mesures de santé publique en présence d'une grippe pandémique. L'annexe des mesures de santé publique renferme des conseils techniques pour les ministères de la Santé fédéral, provinciaux et territoriaux et d'autres ministères qui doivent fournir des services de santé à des populations particulières, comme certaines communautés autochtones et le personnel militaire. Elle souligne l'importance de la collaboration entre tous les ordres du gouvernement, afin de veiller à ce que le public comprenne et adopte les mesures. L'annexe décrit les rôles et responsabilités particuliers des intervenants participant à la sélection et à la mise en œuvre des mesures individuelles et communautaires cherchant à prévenir, à contrôler et à atténuer la grippe pandémique. L'annexe cherche à faciliter l'adoption d'une approche commune en matière de mesures de santé publique à l'échelle des territoires de compétence (2).

Le Groupe de travail sur la préparation du Canada en cas de grippe pandémique (PCGP) a mis à jour l'annexe, afin d'intégrer l'expérience acquise lors de la grippe pandémique H1N1 en 2009, et de tenir compte des objectifs du Canada en matière de pandémie, comme le décrit le document PCGP : "Tout d'abord, réduire au minimum la morbidité grave et la mortalité en général, et deuxièmement réduire au minimum les perturbations sociales » (2). Le document PCGP est un document d'orientation évolutif. L'annexe sera mise à jour dès que la publication de nouveaux renseignements l'exigera. L'annexe devrait être lue en conjonction avec le document principal et les autres annexes techniques du document PCGP, puisqu'ils doivent tous être utilisés ensemble.

Le présent article résume la stratégie relative aux mesures de santé publique en cas de pandémie au Canada, comme l'indique
I'annexe du document PCGP réservée aux mesures de santé publique récemment mise à jour (2). Il fait partie d'une série de documents décrivant l'approche du Canada en matière de préparation en cas de grippe pandémique publiés dans le Relevé des maladies transmissibles au Canada (3-8).

\section{Objectifs de la stratégie relative aux mesures de santé publique du Canada en cas de pandémie}

La stratégie relative aux mesures de santé publique cherche à soutenir les objectifs du Canada en matière de pandémie, en :

- Réduisant la transmission de manière générale et en ralentissant le taux de transmission du nouveau virus ou du virus pandémique, diminuant ainsi le nombre de cas extrêmement graves et de décès, et dispersant I'accumulation de cas au fil du temps

- Réduisant la demande de pointe dans les établissements de soins de santé, afin de les protéger des perturbations sociétaires et du débordement des services communautaires, et de gagner du temps jusqu'à ce qu'un vaccin soit offert

\section{Contexte canadien}

En présence d'une grippe pandémique, il est essentiel que tous les ordres du gouvernement adoptent l'approche en matière de sélection et d'utilisation des mesures de santé publique pour assurer la réussite de l'intervention. Au Canada, les provinces, les territoires et les organismes de santé publique locaux ont le pouvoir de mettre en place des mesures de santé publique dans leurs territoires de compétence. Le gouvernement fédéral est chargé des mesures relatives à la frontière internationale et aux déplacements. II doit, en outre, recommander des mesures de santé publique pour des populations particulières bénéficiaires de programmes et de services de santé fédéraux, comme certaines communautés autochtones et les détenus d'établissements correctionnels.

Le Canada est diversifié en termes de langue, de croyances religieuses, d'ethnicité, de culture et de style de vie. Dans certains cas, les services existants ne permettent pas de combler entièrement les besoins de la population. Le document PCGP souligne que les planificateurs des services de santé doivent adapter les approches aux individus incapables d'accéder aux ressources habituelles et de les utiliser, et dont la situation peut influer sur leur vulnérabilité en cas de grippe pandémique (comme les personnes ayant des incapacités physiques ou mentales, de faibles capacités de lecture et d'écriture, une compréhension limitée de l'anglais ou du français ou souffrant d'insécurité du revenu). 
Il est aussi important d'adapter les approches en fonction de la situation (contexte social, environnemental, économique et accès aux soins de santé) des personnes vivant dans des communautés éloignées et isolées lors de la planification en cas de pandémie. L'annexe énumère les défis uniques auxquels certaines communautés font face ( $p$. ex. un accès limité à des fournitures autres que médicales, comme le savon, la nourriture et les produits ménagers), ainsi que des stratégies potentielles pour mettre en œuvre des mesures de santé publique dans ces communautés ( $p$. ex. le fait d'accroître la sensibilisation des individus et de la communauté, et la nécessité d'avoir une capacité de mobilisation pour ces produits de base).

\section{Principaux éléments de la stratégie relative aux mesures de santé publique}

Les mesures de santé publique sont les mesures les plus fondamentales qui peuvent être prises pour réduire la transmission du virus de la grippe pandémique dans la communauté. Les mesures appliquées lors d'une grippe pandémique se fondent sur les pratiques actuelles pour les maladies respiratoires infectieuses, modifiées et accrues en fonction de l'épidémiologie du virus. Au cours d'une pandémie, les mesures de santé publique sont mises en œuvre par de nombreuses personnes dans différents contextes. C'est pourquoi le contexte dans lequel elles sont appliquées est important.

Le document PCGP fait état de facteurs déclencheurs pour la prise de mesures, comme l'application des mesures de santé publique, en fonction du niveau d'activité du virus pandémique au Canada. On s'attend à ce que la sélection des mesures de santé publique et le moment de leur application varieront en fonction des territoires de compétence, puisque l'activité du virus pandémique différera d'une région à l'autre en ce qui concerne le moment de l'apparition de la vague pandémique et de son intensité.

Parmi les facteurs qui ont un effet sur l'efficacité des mesures de santé publique lors d'une intervention en cas de pandémie, il y a l'épidémiologie de la pandémie (c.-à-d. les caractéristiques du virus), le moment de la mise en œuvre, l'approche quant à leur utilisation (seules ou en combinaison) et la conformité du public. Les mesures doivent être évolutives, souples et proportionnelles à la menace pandémique, afin d'optimiser leur efficacité.

\section{Mesures individuelles}

Au cours d'une pandémie, les mesures de protection personnelles protègent les individus, leurs familles et leurs communautés. Ces mesures sont à la base d'une bonne pratique en santé publique en ce qui concerne la grippe et $d$ 'autres maladies respiratoires. Elles sont systématiquement recommandées. La majorité de ces mesures s'appliquent à tout scénario de pandémie, sans égard aux répercussions attendues (allant du niveau faible à élevé), en fonction du degré de transmissibilité du virus et de gravité clinique.

Parmi les mesures individuelles, il y a les suivantes:
- L'hygiène des mains (c.-à-d. le lavage ou l'antisepsie des mains)

- L'étiquette respiratoire (c.-à-d. se couvrir la bouche et le nez en cas d'éternuements et de toux)

- Le nettoyage et la désinfection des surfaces communément utilisées dans le milieu environnant

- Le port de masques chirurgicaux par les personnes atteintes de la grippe

- L'auto-isolement volontaire (c.-à-d. que la personne se tient à l'écart des autres du moment où les symptômes de la grippe sont reconnus et jusqu'à au moins 24 heures après la disparition des symptômes)

- La quarantaine volontaire à la maison (c.-à-d. que la personne exposée reste à la maison du moment où elle est initialement exposée jusqu'à trois jours après sa dernière exposition)

L'auto-isolement et la quarantaine à la maison peuvent avoir des conséquences secondaires imprévues. Par exemple, certains employeurs peuvent faire preuve d'intolérance envers les absences et la personne malade peut perdre un revenu si le congé n'est pas rémunéré. L'annexe propose des stratégies possibles pour relever ces défis.

\section{Mesures communautaires}

Par mesures communautaires, on entend des stratégies de contrôle des maladies cherchant à réduire et à ralentir la transmission de la grippe dans les communautés. Les autorités de santé publique locales prendront probablement les décisions relatives à la mise en œuvre de ces mesures, en coordination avec les organismes gouvernementaux provinciaux, territoriaux ou régionaux, afin de veiller à l'adoption d'une approche uniforme. L'application des mesures communautaires dépendra du scénario concernant les répercussions de la pandémie, ainsi que du contexte local. Parmi les mesures communautaires, il y a les suivantes:

- Le nettoyage des espaces publics, conformément aux pratiques habituellement adoptées avant la pandémie (p. ex. les produits de nettoyage utilisés, les surfaces nettoyées)

- Les mesures d'isolement social qui limitent la fréquence et la durée des contacts étroits entre des personnes de tout âge dans des contextes de rassemblement de gens, comme les milieux de travail, les garderies, les écoles, les refuges, les lieux de culte ou culturels et les lieux de rassemblement de masse (p. ex. des concerts, des événements sportifs)

Les mesures d'isolement social peuvent avoir des conséquences secondaires imprévues chez les individus, les familles et les communautés, comme la perte de revenu, la nécessité accrue d'obtenir des services de soutien, et l'accès possiblement réduit à certains services. Dans le cadre d'un scénario de pandémie dont les répercussions sont d'un niveau inférieur, les avantages associés à certaines mesures de communautaires pour lutter contre les infections (p. ex. la fermeture proactive 
des écoles) pourraient ne pas être neutralisés par les coûts et les perturbations sociétaires attribuables à ces mesures. II est donc probable que ces mesures seront mises en œuvre uniquement dans le cadre d'un scénario de pandémie à répercussions plus importantes ou dans certaines situations dans quelques communautés.

\section{Mesures relatives aux déplacements et à la frontière}

Parmi les mesures d'intervention en cas de nouvelle grippe pandémique, il y a l'application de mesures de santé publique ciblant les voyageurs internationaux arrivant au Canada ou quittant le pays. Ces mesures se fondent sur les programmes et procédures fédéraux existants. Parmi ces mesures, il y a la prestation de renseignements, la délivrance d'avis sur la santé des voyageurs, et l'administration de la Loi sur la mise en quarantaine (9) à tous les points d'entrée internationaux (p. ex. la mise en application de mesures de contrôle à l'arrivée ou au départ des voyageurs internationaux ou modes de transport). Au moment d'une pandémie, de nombreux facteurs influeront sur les décisions au sujet des mesures à la frontière, y compris les caractéristiques du virus (p. ex. la transmissibilité, la virulence et les facteurs de risque), les preuves de l'efficacité des mesures, ainsi que les risques pour les voyageurs et le public.

Conformément à la Loi sur la mise en quarantaine (9), tous les voyageurs malades doivent faire l'objet d'une détection à l'arrivée à la frontière du Canada ou à leur départ. Présentement, il est difficile de déterminer les cas de nouveau virus de la grippe ou de la grippe pandémique au point d'entrée, en raison de la similitude des symptômes de grippe et de différents autres virus respiratoires. Le contrôle au départ est plus efficace que celui à l'arrivée pour diminuer la transmission de la grippe, parce qu'il réduit le nombre de voyageurs malades embarquant dans un moyen de transport.

\section{Mesures de gestion des cas et des contacts}

Lorsqu'un nouveau virus de la grippe ou de la grippe pandémique est décelé pour la première fois au Canada ou ailleurs, les activités en santé publique qui cherchent à prévenir ou à limiter la propagation du virus au Canada sont entamées. Dans certaines situations, il peut être nécessaire d'assurer une gestion en santé publique des cas d'un nouveau virus de la grippe, ainsi que des contacts étroits (p. ex. la surveillance de la santé, la prestation de conseils sur le moment où il faut consulter un médecin).

La gestion des cas et des contacts par les autorités de santé publique sera probablement assurée de manière plus dynamique au début d'une pandémie, avant qu'il y ait transmission soutenue, afin d'empêcher ou de retarder la propagation du virus. Si la transmission du virus se poursuit, les activités de gestion seront axées sur l'éducation du public, au lieu de la gestion individuelle, afin de renforcer les mesures individuelles et communautaires.

\section{Éducation du public}

En cas de grippe pandémique, la demande du public en matière d'information devrait être extrêmement élevée et être maintenue tandis que la maladie se propage à l'échelle du Canada et dans les communautés locales. Au début d'une pandémie, avant qu'un vaccin soit offert, les mesures de santé publique sont le principal moyen servant à ralentir la transmission. Les campagnes d'éducation du public récentes, s'appuyant sur une approche de communication des risques, sont essentielles pour promouvoir et soutenir la mise en œuvre et l'adoption de mesures chez les individus et dans les communautés. Les stratégies, interventions et produits créés dans le cadre de campagnes de lutte contre la grippe saisonnière peuvent servir de fondements pour les campagnes d'information sur une pandémie. Les messages devront être adaptés pour joindre les personnes dont les besoins ne sont pas satisfaits par les services habituels ou qui sont incapables d'accéder aux ressources, ce qui peut accroître leur vulnérabilité lors d'une pandémie.

\section{Approche de gestion de risque}

La stratégie relative aux mesures de santé publique du Canada fait face à de nombreux risques, y compris la possibilité que les mesures aient des conséquences secondaires imprévues et de I'incertitude quant à l'adoption adéquate de ces mesures par le public. L'annexe comprend l'approche de gestion de risque en PCGP, à l'appui d'une planification évolutive et souple en cas de pandémie, en déterminant les risques et en proposant des mesures d'atténuation. Des communications récentes et transparentes sur les risques pour le public et les fournisseurs de soins de santé devraient faire partie intégrante de l'intervention lors de chaque événement. Le tableau 1 illustre comment I'approche de gestion de risque en PCGP est appliquée à la stratégie relative aux mesures de santé publique.

\section{Tableau 1 : Risques ayant un effet sur la stratégie relative aux mesures de santé publique, leurs répercussions et les mesures d'atténuation ou $d^{\prime}$ 'intervention potentielles}

\begin{tabular}{|c|c|c|}
\hline $\begin{array}{l}\text { Facteur/ } \\
\text { événement }\end{array}$ & Répercussions & $\begin{array}{c}\text { Atténuation/ } \\
\text { intervention } \\
\text { potentielle }\end{array}$ \\
\hline $\begin{array}{l}\text { Les médias signalent } \\
\text { l'apparition d'une } \\
\text { maladie grave ou la } \\
\text { présence d'un nombre } \\
\text { élevé de cas }\end{array}$ & $\begin{array}{l}\text { Il y a une hausse } \\
\text { soudaine de la demande } \\
\text { d'information au sujet de } \\
\text { l'efficacité des mesures } \\
\text { de santé publique } \\
\text { Il faut mettre en œuvre } \\
\text { un nombre supérieur } \\
\text { de mesures de santé } \\
\text { publique (ou peut- } \\
\text { être des mesures plus } \\
\text { ciblées) }\end{array}$ & $\begin{array}{l}\text { Communiquer et accroître } \\
\text { l'éducation du public } \\
\text { concernant les mesures } \\
\text { de santé publique } \\
\text { individuelles et les } \\
\text { raisons connexes (comme } \\
\text { I'hygiène des mains, } \\
\text { l'étiquette en matière } \\
\text { de respiration, l'auto- } \\
\text { isolement volontaire } \\
\text { en cas de maladie, le } \\
\text { nettoyage du milieu } \\
\text { environnant, les soins aux } \\
\text { malades, la consultation } \\
\text { d'un médecin). Utiliser } \\
\text { des approches adaptées } \\
\text { pour communiquer } \\
\text { avec les populations } \\
\text { vulnérables }\end{array}$ \\
\hline
\end{tabular}


Tableau 1 (suite) : Risques ayant un effet sur la stratégie relative aux mesures de santé publique, leurs répercussions et les mesures d'atténuation ou d'intervention potentielles

\begin{tabular}{|c|c|c|}
\hline $\begin{array}{c}\text { Facteur/ } \\
\text { événement }\end{array}$ & Répercussions & $\begin{array}{c}\text { Atténuation/ } \\
\text { intervention } \\
\text { potentielle }\end{array}$ \\
\hline $\begin{array}{l}\text { Les médias signalent } \\
\text { l'apparition d'une } \\
\text { maladie grave ou } \\
\text { la présence d'un } \\
\text { nombre élevé de cas } \\
\text { (suite) }\end{array}$ & $\begin{array}{l}\text { Il y a une hausse } \\
\text { soudaine de } \\
\text { la demande } \\
\text { d'information au sujet } \\
\text { de l'efficacité des } \\
\text { mesures de santé } \\
\text { publique } \\
\text { Il faut mettre en } \\
\text { œuvre un nombre } \\
\text { supérieur de mesures } \\
\text { de santé publique } \\
\text { (ou peut-être des } \\
\text { mesures plus ciblées) } \\
\text { (suite) }\end{array}$ & $\begin{array}{l}\text { Expliquer au public } \\
\text { comment chaque } \\
\text { mesure additionnelle } \\
\text { augmente la } \\
\text { protection individuelle } \\
\text { et du groupe et la } \\
\text { prévention } \\
\text { Informer le public } \\
\text { que les mesures } \\
\text { peuvent changer } \\
\text { lorsque de nouveaux } \\
\text { renseignements } \\
\text { deviennent } \\
\text { accessibles }\end{array}$ \\
\hline $\begin{array}{l}\text { Les différences en } \\
\text { ce qui concerne } \\
\text { la mise en œuvre } \\
\text { des mesures de } \\
\text { santé publique } \\
\text { entre les territoires } \\
\text { de compétence } \\
\text { et à l'échelle } \\
\text { internationale }\end{array}$ & $\begin{array}{l}\text { La sélection et la } \\
\text { mise en œuvre des } \\
\text { mesures de santé } \\
\text { publique varient } \\
\text { en fonction des } \\
\text { contextes locaux ou } \\
\text { régionaux } \\
\text { Le public peut } \\
\text { considérer que } \\
\text { l'approche d'un } \\
\text { autre territoire de } \\
\text { compétence est } \\
\text { meilleure } \\
\text { Le public peut avoir } \\
\text { des préoccupations } \\
\text { s'il perçoit que } \\
\text { les ressources en } \\
\text { santé publique sont } \\
\text { réparties de manière } \\
\text { inéquitable }\end{array}$ & $\begin{array}{l}\text { Reconnaître les } \\
\text { différences en ce } \\
\text { qui concerne les } \\
\text { approches locales } \\
\text { ou régionales et les } \\
\text { expliquer } \\
\text { Veiller à ce que les } \\
\text { mesures de santé } \\
\text { publique soient } \\
\text { bénéfiques pour tous } \\
\text { les groupes d'une } \\
\text { communauté ou } \\
\text { d'une région, et que } \\
\text { le fardeau soit réparti } \\
\text { équitablement }\end{array}$ \\
\hline
\end{tabular}

Source : Conseil du Réseau pancanadien de santé publique. Annexe des mesures de santé publique : Préparation du Canada en cas de grippe pandémique : Guide de planification pour le secteur de la santé (2)

\section{Discussion}

La préparation et l'intervention du Canada en cas de grippe pandémique exigent l'adoption d'une approche à facettes multiples, et les mesures de santé publique représentent un aspect essentiel lors d'une pandémie. Les mesures de santé publique, qui exigent l'aide individuelle et de la communauté pour être mises en place, sont les mesures les plus fondamentales qui peuvent être prises pour réduire la transmission du virus de la grippe pandémique; elles sont déjà utilisées à l'échelle du Canada lors des éclosions de grippe saisonnière et d'autres maladies transmissibles. Les mesures de santé publique cherchent à réduire l'occurrence et la durée des infections humaines, afin de retarder l'atteinte du pic de l'activité grippale pandémique.

Il est important que les planificateurs des services de santé tiennent compte du fait que les mesures de santé publique doivent être mises en œuvre au début d'une pandémie, d'une manière ciblée et stratifiée. Une approche en communication transparente et uniforme peut aider à assurer la confiance du public et l'adoption des mesures recommandées. Il faut évaluer les avantages éventuels des mesures particulières en fonction de la valeur concrète et de la faisabilité de leur mise en œuvre, ainsi que des répercussions sociales et économiques secondaires imprévues potentielles. Les mesures doivent être adaptées aux populations et aux contextes, puisque la situation de chacun peut accroître la vulnérabilité lors d'une grippe pandémique.

\section{Conclusion}

L'orientation expliquée dans l'annexe des mesures de santé publique du document PCGP cherche à promouvoir l'adoption d'une approche évolutive et uniforme en ce qui concerne la planification de la pandémie. Elle peut être adaptée à différents contextes. De nombreuses mesures recommandées dépendent de facteurs déclencheurs locaux; le moment de leur mise en œurre varie donc en fonction de la situation locale qui peut être différente au même moment dans les autres territoires de compétence canadiens. Cependant, I'adoption d'une approche uniforme lors de l'application des mesures de santé publique et des messages connexes permettra d'améliorer la perception du public, d'augmenter sa confiance et d'assurer l'adoption de l'orientation.

\section{Déclaration des auteurs}

Le Groupe de travail sur la préparation du Canada en cas de grippe pandémique est constitué de $B$. Henry (présidente), de C. Alfieri, d'I. Gemmill, de T. Hatchette, d'E. Henry, de S. Hota, d'A. Lebans et de B. Schwartz.

\section{Conflit d'intérêts}

Aucun.

\section{Remerciements}

L'auteure souhaite remercier la Division des programmes d'immunisation et de la préparation aux pandémies de l'Agence de la santé publique du Canada pour sa contribution à la rédaction de ce sommaire : E. Henry (directrice), L. Paddle, 
S. Smith, J. Williams, F. Lalonde et O. Baclic, et S .Rempel, qui a participé à la rédaction de la première ébauche.

\section{Aide financière}

Les travaux du Groupe de travail sur la préparation du Canada en cas de grippe pandémique sont appuyés par l'Agence de la santé publique du Canada.

\section{Références}

1. Conseil du Réseau pancanadien de santé publique. Préparation du Canada en cas de grippe pandémique : Guide de planification pour le secteur de la santé. Ottawa: Agence de la santé publique du Canada; 2018. https://www.canada.ca/fr/sante-publique/ services/grippe-influenza/preparation-canada-cas-gripp e-pandemique-guide-planification-secteur-sante.html

2. Conseil du Réseau pancanadien de santé publique. Mesures de santé publique : Préparation du Canada en cas de grippe pandémique : Guide de planification pour le secteur de la santé. Ottawa : Agence de la santé publique du Canada; 2019. https://www.canada.ca/fr/ sante-publique/services/grippe-influenza/preparation-canad a-cas-grippe-pandemique-guide-planification-secteur-sante/ lignes-directrices-sur-les-mesures-de-sante-publique.html

3. Henry B Gadient $\mathrm{S}$ au nom du Groupe de travail sur la préparation du Canada en cas de grippe pandémique. Stratégie canadienne de vaccination en cas de pandémie. Relevé des maladies transmissibles au Canada 2017;43(7/8):185-9. DOI
4. Henry B au nom du Groupe de travail sur la préparation du Canada en cas de grippe pandémique (GTPCP). Préparation du Canada en cas de grippe pandémique : Stratégie de laboratoire. Relevé des maladies transmissibles au Canada 2018;44(1):11-5. DOI

5. Henry B au nom du Groupe de travail sur la préparation du Canada en cas de grippe pandémique (GTPCP). Préparation du Canada en cas de grippe pandémique : Stratégie de surveillance. Relevé des maladies transmissibles au Canada 2018;44(1):16-20. DOI

6. Henry B au nom du Groupe de travail sur la préparation du Canada en cas de grippe pandémique (GTPCGP). Préparation du Canada en cas de grippe pandémique : Guide de planification pour le secteur de la santé. Relevé des maladies transmissibles au Canada 2018;44(1):7-10. DOI

7. B. Henry au nom du groupe de travail sur le document Préparation du Canada en cas de grippe pandémique (PCGP). Préparation du Canada en cas de grippe pandémique : Stratégie de communication. Relevé des maladies transmissibles au Canada 2018;44(5):118-22. DOI

8. Henry B au nom du Groupe de travail sur la préparation du Canada en cas de grippe pandémique. Préparation du Canada en cas de grippe pandémique : Stratégie antivirale. Relevé des maladies transmissibles au Canada 2019;45(1):41-6. DOI

9. Département de la Justice. Loi sur la mise en quarantaine, SRC 2005, c20, s82. Abroge SRC 1985, cQ-1.

Ottawa : Gouvernement du Canada. (Accédé 2019-03-05). https://laws-lois.justice.gc.ca/fra/lois/q-1.1/page-1.html 